\title{
Intraoperative neuromonitoring with MEPs and prediction of postoperative neurological deficits in patients undergoing surgery for cervical and cervicothoracic myelopathy
}

\author{
Aaron J. Clark, M.D., Ph.D., John E. Ziewacz, M.D., M.P.H., Michael Safaee, B.S., \\ Darryl Lau, M.D., Russ Lyon, M.S., Dean Chou, M.D., Philip R. Weinstein, M.D., \\ Christopher P. Ames, M.D., John P. Clark III, Ph.D., and Praveen V. Mummaneni, M.D.
}

Department of Neurological Surgery, University of California, San Francisco, California

\begin{abstract}
Object. The use of intraoperative neurophysiological monitoring (IONM) in surgical decompression surgery for myelopathy may assist the surgeon in taking corrective measures to reduce or prevent permanent neurological deficits. We evaluated the efficacy of IONM in cervical and cervicothoracic spondylotic myelopathy (CSM) cases.

Methods. The authors retrospectively reviewed 140 cases involving patients who underwent surgery for CSM utilizing IONM during 2011 at the University of California, San Francisco. Data on preoperative clinical variables, intraoperative changes in transcranial motor evoked potentials (MEPs), and postoperative new neurological deficits were collected. Associations between categorical variables were analyzed with the Fisher exact test.

Results. Of the 140 patients, $16(11 \%)$ had significant intraoperative decreases in MEPs. In 8 of these cases, the MEP signal did not return to baseline values by the end of the operation. There were $8(6 \%)$ postoperative deficits, of which 6 were C-5 palsies and 2 were paraparesis. Six of the patients with postoperative deficits had demonstrated persistent MEP signal change on IONM. There was a significant association between persistent MEP changes and postoperative deficits $(\mathrm{p}<0.001)$. The sensitivity of intraoperative MEP monitoring was $75 \%$, the specificity $98 \%$, the positive predictive value $75 \%$, and the negative predictive value $98 \%$. Due to higher rates of false negatives, the sensitivity decreased to $60 \%$ in the subgroup of patients with vascular disease comorbidity. The sensitivity increased to $100 \%$ in elderly patients and in patients with preoperative motor deficits. The sensitivity and positive predictive value of deltoid and biceps MEP changes in predicting C-5 palsy were $67 \%$ and $67 \%$, respectively.

Conclusions. The authors found a correlation between decreased intraoperative MEPs and postoperative new neurological deficits in patients with CSM. Sensitivity varies based on patient comorbidities, age, and preoperative neurological function. Monitoring of MEPs is a useful adjunct for CSM cases, and the authors have developed a checklist to standardize their responses to intraoperative MEP changes.

(http://thejns.org/doi/abs/10.3171/2013.4.FOCUS13121)
\end{abstract}

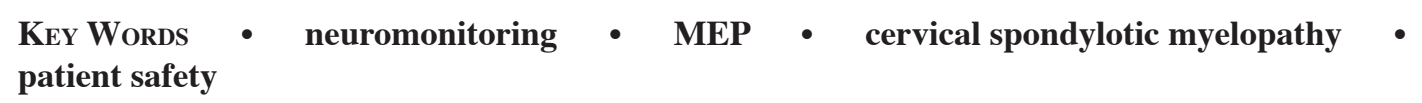

I NTRAOPERATIVE neurophysiological monitoring (IONM) is a rapidly advancing field, and neurophysiological monitoring is becoming prevalent in spinal surgery.5, ${ }^{5,17}$ Although almost universally employed during spinal deformity surgery, the indications in "lower risk" decompressive procedures are more controversial..$^{20}$ The sensitivity of combined-modality monitoring may approach $100 \%$, although sensitivities as low as $43 \%$ have been reported. ${ }^{21}$

\footnotetext{
Abbreviations used in this paper: $\mathrm{CSM}=$ cervical spondylotic myelopathy (including cervicothoracic cases); IONM = intraoperative neurophysiological monitoring; MEP = motor evoked potential; MRC $=$ Medical Research Council; SSEP $=$ somatosensory evoked potential.
}

Somatosensory evoked potentials, although advantageous because they can be monitored continuously, rely on signal averaging over time, and decreases may significantly lag behind transcranial MEP changes. Monitoring of MEPs may provide earlier detection of neurological injury and is associated with high sensitivity. ${ }^{10}$ However, MEPs cannot be monitored continuously, may induce patient movement, and are negatively affected by inhaled anesthetic agents. ${ }^{10}$ Some argue against the use of IONM in cervical decompression altogether. ${ }^{20}$ Few studies have focused on the use of IONM in decompressive surgery for myelopathy., ${ }^{3,12}$ In this study, we analyzed a large group of consecutive cases involving patients with CSM (including patients with cervicothoracic myelopathy in addition to those with purely 
cervical myelopathy) who underwent decompressive surgery at a single center over the course of 1 year with the use of MEP.

\section{Methods}

\section{Data Collection}

Patients were identified by first screening the IONM reports for all operations performed in the University of California, San Francisco, Department of Neurological Surgery from January 1 to December 31, 2011. Hospital records and operative reports of all patients who underwent spine procedures were examined. All patients who underwent cervical or cervicothoracic decompressive operations with IONM for myelopathy due to degenerative spondylosis during that time were included in our study. All patients consented to IONM as part of the surgical informed consent process. Patient demographic data were obtained from hospital and clinic charts. Age was dichotomized into 65 years or more and less than 65 years. Data regarding risk factors for vascular disease were also extracted from hospital charts; the risk factors included morbid obesity, coronary artery disease or history of myocardial infarction, peripheral vascular disease, history of stroke, diabetes, and tobacco use (Table 1). Data on preoperative and postoperative neurological function were extracted from chart documentation of the neurosurgeon's objective examination, including the MRC validated motor scale, sensory disturbance, and evidence of hyperreflexia or pathological reflexes. To capture postoperative neurological deficits, the hospital chart was reviewed until the day of discharge. In addition, the outpatient clinic charts were also reviewed (mean 6 months). Postoperative neurological deficit was defined as new or worsening motor weakness after surgery. IONM alerts were extracted from the IONM report. A significant MEP alert was defined as an abrupt decrease in peak-to-peak amplitude of more than $50 \%$ for more than 3 successive trials over a 1-3 minute period.

\section{IONM MEP Recording}

Transcranial MEPs were generated by multipulse transcranial electrical stimulation $(0-800 \mathrm{~V}, 50-75 \mu \mathrm{sec}$ pulse duration, $0-9$ pulses at $1-3 \mathrm{msec}$ ) delivered to electrodes placed over motor cortical regions at $\mathrm{C} 3$ and $\mathrm{C} 4$ using a Cadwell TCS-4 constant voltage transcranial stimulator. Electromyographic responses were recorded from needle electrodes placed bilaterally in the deltoid (axillary nerve, C5-6), biceps (musculocutaneous nerve, C5-7), triceps (radial nerve, C6-8), and thenar and hypothenar eminences of the hand (C8-TI). Waveforms were recorded on a commercially available Cadwell neurophysiology workstation (model Elite or Cascade).

\section{Statistical Analysis}

A true positive was defined as presence of an IONM MEP alert during surgery followed by a neurological deficit in the postoperative period. Conversely, a true negative was defined as absence of IONM MEP alerts during surgery and lack of a new neurological deficit after
TABLE 1: Demographic characteristics of 140 patients with cervical or cervicothoracic spondylotic myelopathy*

\begin{tabular}{cc}
\hline Characteristic & Value \\
\hline age (yrs) & \\
median & 63 \\
range & $27-90$ \\
$\geq 65$ yrs & $60(43)$ \\
female sex & $41(29)$ \\
spinal level & \\
cervical & $133(95)$ \\
thoracic & $6(4)$ \\
cervicothoracic & $1(1)$ \\
T2 signal abnormality & \\
yes & $71(54)$ \\
missing & $8(6)$ \\
vasculopathy risk factors & \\
yes & $55(39)$ \\
preop motor deficit & \\
yes & $81(58)$ \\
operation & \\
anterior & $47(34)$ \\
posterior & $87(62)$ \\
both & $6(4)$ \\
instrumentation & \\
yes & $134(96)$ \\
laminoplasty & $40(29)$ \\
follow-up (wks) & \\
mean & 25.2 \\
range & $2-66$ \\
\hline & \\
\hline & \\
\hline &
\end{tabular}

* Values represent numbers of patients (\%) unless otherwise indicated.

surgery. A false positive was defined as presence of an IONM MEP alert during surgery that was not followed by a neurological deficit. A false negative was defined as absence of IONM MEP alerts during an operation followed by a new neurological deficit. Sensitivity was calculated as true positives/(true positives + false negatives). Specificity was calculated as true negatives/(true negatives + false positives). Positive predictive value was calculated as true positives/(true positives + false positives). Negative predictive value was calculated as true negatives/ (true negatives + false negatives). Associations between IONM MEP changes and postoperative new neurological deficits were analyzed with the Fisher exact test. All analyses were performed using PASW Statistics 18.0 software (SPSS, Inc.); $p$ values less than 0.05 were considered statistically significant.

\section{Results}

\section{Patient Demographics}

During the 1-year study period, 140 patients underwent decompressive surgery with IONM for myelopathy. Their median age was 63 years, and $71 \%$ of the patients were 
male. In 54\% of cases, T2 signal abnormality was noted on preoperative MR images. All patients had evidence of spinal cord compression on MRI, and 58\% had a preoperative motor deficit, defined as less than $5 / 5$ motor function in any single muscle group on the MRC scale. Anterior fusion was performed in 34\% of cases, posterior fusion in $34 \%$, laminoplasty in $29 \%$, and laminectomy in $3 \%$.

\section{Intraoperative MEP Changes and Outcome}

Major IONM MEP alerts were recorded in 16 operations (11\%). In 8 cases, the MEP signal did not return to baseline. Overall, 8 patients $(6 \%)$ experienced new postoperative neurological deficits. Six patients had a C-5 palsy, one had bilateral arm weakness, and one had bilateral leg weakness. In those with postoperative deficits, the median MRC strength was 3/5 (range 1-4). There was a significant association between IONM MEP alerts and the presence of a new postoperative deficit (Table 2, $p$ $<0.001$ ). This corresponds to a sensitivity of $75 \%$ and a specificity of $92 \%$. The positive predictive value of IONM MEP alerts was $38 \%$, and the negative predictive value was $98 \%$. Specificity (98\%) and positive predictive value (75\%) were higher for MEP changes that were not followed by return to baseline (Table 3 ).

\section{Vascular Disease Risk Factors, MEP Changes, and Outcome}

We were interested in identifying specific groups of patients who might have higher associations between MEP changes and postoperative deficits. Fifty-five patients $(39 \%)$ had risk factors for vascular disease. Of these, 17 had coronary artery disease, 19 had diabetes mellitus, 17 used tobacco products, 12 were morbidly obese, and 6 had peripheral vascular disease. Many patients had more than one risk factor. Within this subgroup of patients, there was a higher rate of neuromonitoring changes in those with a postoperative deficit compared with those without one (Table $4, p=0.001$ ). However, this corresponds to a lower sensitivity $(60 \%)$ than in the overall patient group. In the remaining patients with no vascular risk factors, the sensitivity was higher (100\%).

\section{Preoperative Motor Deficit, MEP Changes, and Outcome}

Eighty-one patients $(58 \%)$ presented with a preexisting motor deficit. In this subgroup, MEP changes were

TABLE 2: Contingency table describing the association between IONM MEP alerts at any time during the operation and new postoperative neurological deficits*

\begin{tabular}{ccc}
\hline \multicolumn{1}{c}{ Variable } & MEP Alert & No Alert \\
\hline no. of cases & 16 & 124 \\
new motor deficit & & \\
yes (\%) & $6(38)$ & $2(2)$ \\
no (\%) & $10(62)$ & $122(98)$ \\
\hline
\end{tabular}

* There was a significant association between MEP alert and new deficit $(p<0.001)$. Maneuvers were done based on the IONM checklist described by Ziewacz et al. ${ }^{22}$ that may have resulted in resolution of some of the alerts.
TABLE 3: Contingency table describing the association between IONM MEP alerts that persisted until completion of the operation and new postoperative neurological deficits*

\begin{tabular}{ccc}
\hline \multicolumn{1}{c}{ Variable } & MEP Alert & No Alert \\
\hline no. of cases & 8 & 132 \\
new motor deficit & & \\
yes $(\%)$ & $6(75)$ & $2(1.5)$ \\
no $(\%)$ & $2(25)$ & $130(98.5)$ \\
\hline
\end{tabular}

* Significant association between MEP alert and new deficit $(p<0.001)$.

significantly associated with new or worsening postoperative motor deficits (Table $4, \mathrm{p}<0.001$ ). The sensitivity was $100 \%$ and the specificity was $99 \%$. The positive predictive value was $80 \%$, and the negative predictive value was $100 \%$. In patients who did not have motor deficits before surgery, the sensitivity was lower (50\%).

\section{Age, MEP Changes, and Outcome}

In the study group, there were 60 patients (43\%) who were 65 years of age or older. In this subgroup, there was also an association between IOMN MEP alerts and postoperative deficits (Table $4, \mathrm{p}<0.001$ ). The sensitivity was $100 \%$, and the specificity was $98 \%$. The positive predictive value was $80 \%$, and the negative predictive value was $100 \%$. In patients less than 65 years of age, the sensitivity was lower (50\%).

\section{Changes in Deltoid/Biceps MEP and Postoperative C-5 Palsy}

Changes in deltoid or biceps MEPs were observed in 8 cases. In 6 cases (4\%) the MEP signal did not return to baseline by the completion of the operation. There was a significant association between MEP changes without return to baseline and postoperative $\mathrm{C}-5$ palsy (Fig. 1, Table $5, \mathrm{p}<0.001)$. The sensitivity was $67 \%$, the specificity was $99 \%$, the positive predictive value was $67 \%$, and the negative predictive value was $99 \%$. We also compared the rate of postoperative $\mathrm{C}-5$ palsy in patients who underwent only posterior approaches and those who underwent only anterior approaches. Although there was a trend toward an increased rate of C-5 palsy after posterior-only operations (5.7\% vs $2.1 \%$ for anterior), it was not statistically significant $(\mathrm{p}=0.67)$.

\section{Discussion}

Intraoperative neurophysiological monitoring is routinely employed in spine surgery, particularly for procedures involving spinal neoplasms, spinal vascular lesions, and scoliosis correction. Over the past decade, IONM has also been incorporated into surgical procedures involving the cervical spine with increasing frequency. ${ }^{5,9,17}$ However, there continues to be significant debate regarding its utility in predicting and mitigating postoperative neurological deficits in cervical spine surgery. ${ }^{1,11,13,14,20,21}$ Considerations against the use of IONM for cervical spine surgery include the additional cost and difficulty interpreting marginal 
TABLE 4: Association between persistent IONM MEP alerts and new postoperative neurological deficits in different subgroups of patients with CSM

\begin{tabular}{ccccc}
\hline New Deficit & Subgroup & MEP Alert & No Alert & p Value \\
\hline yes $(\%)$ & vascular risk factors & $3(75)$ & $2(4)$ & 0.001 \\
no $(\%)$ & & $1(25)$ & $49(96)$ & \\
yes $(\%)$ & preop deficits & $4(80)$ & $0(0)$ & $<0.001$ \\
no $(\%)$ & & $1(20)$ & $76(100)$ & $<0.001$ \\
yes $(\%)$ & age $\geq 65$ yrs & $4(20)$ & $0(0)$ & \\
no $(\%)$ & & $1(80)$ & $55(100)$ & \\
\hline
\end{tabular}

waveform changes..$^{12,20}$ As rising health care costs become a greater focus, minimizing cost while not compromising patient care is critical. Traynelis et al. ${ }^{20}$ performed a retrospective cost analysis of 720 cases in which patients underwent anterior, posterior, or combined anterior-posterior cervical spine surgery without IONM. They concluded that decompression and reconstruction/fusion for symptomatic cervical spine disease without IONM may reduce the cost of treatment without adversely impacting patient safety. Their rationale stemmed from low rates of postoperative deficits $(0.4 \%)$ and an estimated additional cost of more than a million dollars in their series of patients who had undergone IONM. A major limitation to their cost analysis was estimating that each procedure required an IONM average of 4 hours; this may be an overestimate for common procedures such as single-level anterior cervical discectomy and fusion, anterior cervical corpectomy and fusion, and laminectomy. In addition, the comparative costs of mitigating future events versus managing postoperative
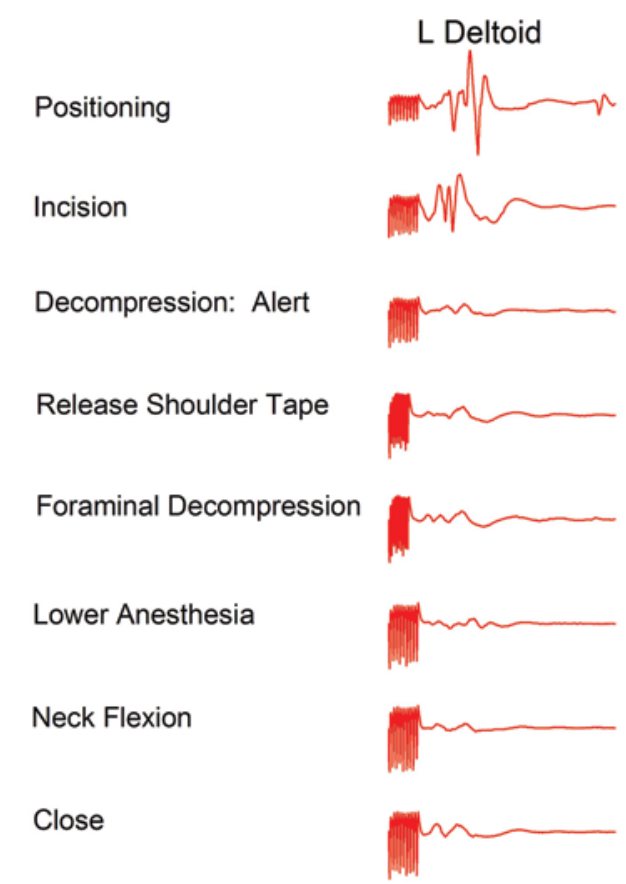

neurological deficits that may have been prevented with IONM are unknown. Based on the study by Traynelis et al. ${ }^{20}$, the cost of IONM in 2011 based on Medicare billing is $\$ 158.33$ /hour with an additional flat fee of $\$ 233.26$ and \$247.69 for upper- and lower-extremity MEPs, respectively. The cost of neurological injury, particularly spinal cord injury, is extremely high. Ney et al. ${ }^{16}$ recently reported cost-effectiveness data with respect to all spinal surgery and demonstrated a savings of $\$ 63,387$ per neurological injury averted. However, it has not yet been definitively proven that IONM can help prevent neurological injury during surgery.

There are known limitations to IONM when using SSEPs or MEPs. Multiple nonneurological related factors can affect the waveforms for both of these modalities; among these are blood pressure (mean and diastolic), heart rate, temperature, partial pressure of alveolar carbon dioxide, and anesthetic drugs. ${ }^{20}$ In addition, challenging clinical conditions, such as severe myelopathy, spinal cord tumor,
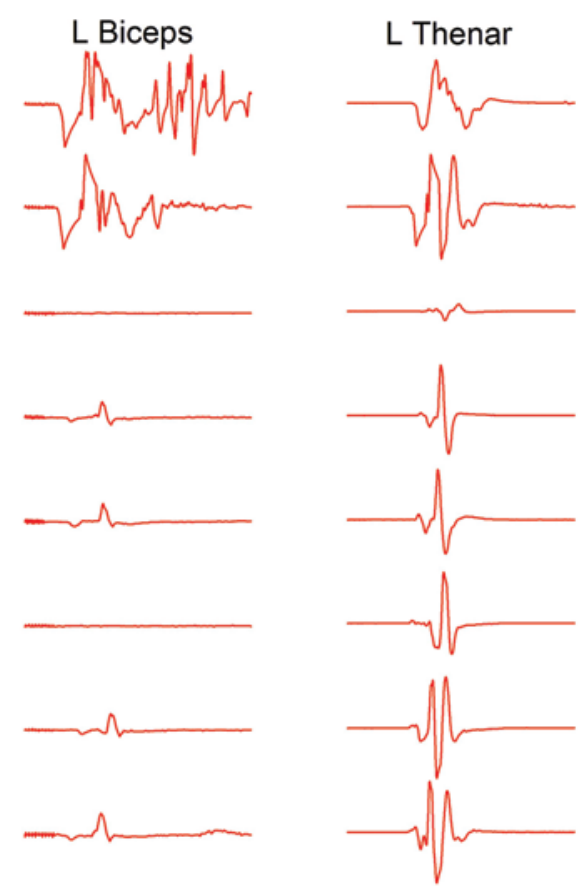

FIG. 1. Representative intraoperative MEP waveform recordings obtained in a 79 -year-old man with bilateral upper-extremity weakness and difficulty ambulating who underwent a C3-4 and C4-5 anterior cervical discectomy and fusion for C3-5 CSM. During the decompression, there was loss of MEP signal from the deltoid, biceps, and thenar muscles. The biceps and deltoids did not recover by completion of the case, despite the intraoperative measures that were taken. The patient was noted to have a $\mathrm{C}-5$ palsy upon awakening as predicted by MEP monitoring. $\mathrm{L}=$ left. 
TABLE 5: Association between IONM MEP alerts in the deltoid and/or biceps without return to baseline and new or worsened C-5 palsy postoperatively*

\begin{tabular}{ccc}
\hline Variable & MEP Alert & No Alert \\
\hline no. of cases & 6 & 134 \\
new C-5 palsy & & \\
yes $(\%)$ & $4(67)$ & $2(1.5)$ \\
no $(\%)$ & $2(33)$ & $132(98.5)$ \\
\hline
\end{tabular}

* Significant association between MEP alert and new deficit $(p<0.001)$.

obesity, or peripheral neuropathy, can make interpretation difficult or even impossible. ${ }^{18}$ An example presented by Bose et al. ${ }^{1}$ involved a patient with preexisting upperextremity weakness who subsequently developed quadriparesis postoperatively. MEP monitoring was precluded by the use of excessive neuromuscular blockade during the procedure, and the patient's baseline posterior tibial nerve SSEPs were severely attenuated, precluding reliable monitoring. Additionally, despite relatively high reported rates of sensitivity and specificity for IONM, postoperative neurological deficits may also arise in cases without significant changes in monitoring signals..$^{2,4,19}$

Despite concerns regarding the cost and functionality of IONM, the preponderance of the current literature concentrating on this topic supports the use of IONM, and some authors demonstrated significant benefits in being able to prevent spinal cord injury and/or postoperative neurological deficits. ${ }^{1,3,6,7,10,11,14,21}$ As we have shown in this study, intraoperative decreases in MEPs were significantly associated with new postoperative deficits following cervical decompression for myelopathy. Other studies have also found similar results. Bose et al. ${ }^{1}$ and Xu et al. ${ }^{21}$ both demonstrated that intraoperative SSEP and MEP monitoring are useful and correlative techniques for identifying changes in spinal cord function and preventing neurological deficits, respectively. Bose et al. performed a retrospective review of 119 cases involving patients who underwent instrumented anterior cervical spine surgery (the majority of patients had only radiculopathy) and found that neurophysiological alerts were present more often during multilevel decompression. In addition, they noted that certain physiological changes and maneuvers resulted in alerts as well: sudden decreases in blood pressure (detecting hypotensive effects on the spinal cord), unusual arm positioning, and hyperextension of the neck. Xu et al. ${ }^{21}$ retrospectively reviewed 57 cases involving patients who underwent anterior cervical discectomy and fusion with SSEP and MEP monitoring. In their series, they emphasized that intraoperative SSEP and MEP monitoring allowed them to immediately make changes in the operative procedure and implement measures that protect the spinal cord (such as maintaining adequate blood pressure and intravenous administration of steroid agents). This occurred in 5\% of their patients. Permanent neurological deficits did not develop in any of these patients; ${ }^{21}$ however, there was no control group for comparison. The authors suggested that MEP monitoring is an effective modality in anterior cervical spine surgery given the anterior location of the corticospinal tracts and the fact that MEP monitoring relies on these tracts.

Whereas Bose et al. ${ }^{1}$ and $\mathrm{Xu}$ et al. ${ }^{21}$ concentrated on patients who underwent anterior cervical spine surgery, most of the patients in our cohort underwent posterior decompression. Prior studies suggested that IONM offers significant benefits to patients undergoing posterior cervical spine surgery as well, especially when utilizing MEPs. ${ }^{3,11}$ In our study we found that MEP alerts in the biceps and deltoid distribution were associated with postoperative C-5 palsies. A similar observation was reported in a study by Fan et al. ${ }^{6}$ In patients undergoing cervical laminectomy, C-5 palsy is not uncommon given the horizontal exit of the C-5 spinal nerve from the vertebral column and its relatively short length compared with other spinal nerves, both of which make it more susceptible to stretch injuries. Therefore utilization of MEPs in posterior cervical decompression may be beneficial in preventing postoperative deficit, especially C-5 palsy. Our study also included corpectomy and nonfusion (laminoplasty) procedures, which we have previously reported have similar risks of C-5 palsy. ${ }^{8,15}$

Overall, significant abnormal alerts occur in $3 \%$ to $12 \%$ of patients undergoing cervical spine surgery with IONM.1,10-12,14,21 This range is likely due to differences in study definitions of an abnormal signal or waveform, which in turn also affect the performance (including sensitivity and specificity) of IONM as a diagnostic test. In addition, the ability to interpret indistinguishable waveforms can be limited because of small amplitudes and poor waveform morphology. ${ }^{1}$ This factor also influences the test performance of IONM. Nonetheless, the performance measures for MEP monitoring in conjunction with SSEP monitoring have been reported as high-sensitivity $(100.0 \%)$, specificity $(95.6 \%)$, positive predictive value (75.0\%), and negative predictive value (100\%) - and superior to SSEP monitoring alone. ${ }^{21}$

While we assessed the utility of using MEPs alone, our results demonstrated a high specificity (92\%) and negative predictive value (98\%), with $75 \%$ sensitivity and a low positive predictive value (38\%). Although SSEP monitoring and MEP monitoring are different modalities of IONM, they are commonly used in conjunction with one another. One possibility for the low positive predictive value in our study is that in our institution many steps are undertaken to mitigate possible spinal cord injury following a significant MEP alert, which might possibly have prevented neurological injury. However, there is no control group available for comparison. Studies comparing MEP and SSEP monitoring directly have suggested that MEP monitoring is superior to SSEP monitoring. A study of 427 patients who underwent cervical spine surgery demonstrated that MEP monitoring was able to identify all patients who experienced new postoperative deficit (100\% sensitivity) with no false positives (100\% specificity). ${ }^{10}$ In the same study, the sensitivity for SSEP monitoring was only $25 \%$. Kim et al. ${ }^{12}$ reported similar rates of sensitivity and specificity with MEPs among their cohort as well (100\% and $90 \%$, respectively). In addition, MEP monitoring may lead to earlier detection of changes than SSEP monitoring, possibly allowing earlier interventions to be implemented. ${ }^{10}$ 


\section{Checklist for Neuromonitoring (MEP) Alert in Patients with Myelopathy or Deformity}

\section{Spine Surgeon:}

Stop current manipulation

Assess field for structural cord compression (misplaced hardware or bone graft, osteophytes, or hematoma)

Perform further decompression if stenosis is present

Consider reversing correction of a spinal deformity

\section{Neurophysiologist:}

Repeat trials of MEPs and SSEPs to rule out potential false positive

Check all leads to make sure no pull-out, may add leads in proximal muscle

groups if possible

Assess the pattern of changes

Asymmetric changes (associated with cord or nerve root injury)

Symmetric changes (associated with anesthetic or hypotension issues)

Quantify improvement and communicate to the surgical team

\section{Anesthesiologist:}

Check if neuromuscular blockade (muscle relaxant) given

If yes, $\square$ Check train of four (TOF)

Verify that no change in anesthetic administration occurred

Assess anesthetic depth
BP
RR
HR
BIS monitor (if available)

Restore or maintain blood pressure (goal mean arterial pressure of 90-100)

Check Hemoglobin/Hematocrit (goal hemoglobin >9-10)

Check temperature and I/O's for adequate resuscitation

Check extremity position in case of plexus palsy

Lighten depth of anesthesia

Reduce to $1 / 3 \mathrm{MAC}$ or temporarily eliminate inhaled agents (i.e. desflurane)

Reduce intravenous anesthetics such as propofol (which may accumulate

systemically during the case and blunt MEPs)

Add adjuvant agents such as Ketamine to permit reduction of MEP

suppressive agents (i.e. propofol and inhalational anesthetics)

\section{IF No Change:}

Increase MAP >100

Consider Steroid Administration

Consider Wake-up test

Consider Aborting surgery

Consider Calcium Channel Blocker (topical to cord or iv)

*The checklist assumes baseline anesthetic regimen is 1/3-1/2 MAC of halogenated anesthetic (desflurane) and TIVA

(total intravenous anesthesia) with propofol $+/$ - ketamine.

FIG. 2. Checklist for the response to an IONM alert. BIS = bispectral index, $B P=$ blood pressure; $H R=$ heart rate; $I / O=$ input/ output; iv = intravenous; $\mathrm{MAC}=$ minimum alveolar concentration; MAP = mean arterial pressure; $\mathrm{RR}=$ respiration rate. Reproduced with permission from Ziewacz et al: Neurosurg Focus 33(5):E11, 2012.

While there is evidence to suggest that MEP monitoring is superior to SSEP monitoring, other studies have emphasized the importance of utilizing both SSEPs and MEPs in detecting neurological compromise, spinal cord injury, and potential postoperative deficits. ${ }^{6,11}$ In a partially retrospective, partially prospective study of 200 patients who underwent cervical laminectomy and laminoplasty, the authors demonstrated that, with SSEP moni- 
toring alone, 6 of 6 patients who developed C-5 palsy experienced this complication despite unremarkable SSEP monitoring. ${ }^{11}$ However, when they prospectively added in MEP monitoring with SSEP monitoring, 2 of 2 cases of postoperative C-5 palsy were accurately predicted. In addition, a prospective study of 1055 patients who underwent cervical spine surgery (of any type) with SSEP ( \pm MEP) monitoring concluded not only that IONM is helpful in predicting and preventing neurological deficit, but also that utilizing combined IONM methods can increase sensitivity significantly. ${ }^{6}$ Although our study focused on MEPs alone, the best modality, or combination of modalities remains to be determined.

A principal finding from this study was that diagnostic performance of MEP monitoring depended on certain risk factors among patients. Patients with vascular risk factors had low MEP sensitivity $(60 \%)$, but in patients without vascular disease the sensitivity was $100 \%$. In addition, MEP monitoring in patients older than 65 years had a sensitivity of $100 \%$, specificity of $98 \%$, positive predictive value of $80 \%$, and negative predictive value of $100 \%$. Other reported risk factors that influence MEP monitoring performance, including obesity and increased length of surgery, may result in false positives. ${ }^{12}$ Therefore, selecting patients who might benefit most from MEP monitoring will likely result in higher sensitivity and specificity, while partly mitigating the increase in cost.

A potential advantage of utilizing IONM-based on SSEPs, MEPs, or both-is to adequately implement immediate actions to prevent spinal cord injury and postoperative neurological deficits in the event of a significant monitoring change. In this light, based on a large retrospective study of 1445 cases, Lee et al. ${ }^{14}$ recommended the following when an IONM alert is encountered: reverse any antecedent surgical event (for example, distraction or neck extension.), maintain mean arterial blood pressure between 85 and $95 \mathrm{~mm} \mathrm{Hg}$, and if there is a partial or complete return of IONM signal within 20 minutes to continue on with surgery. If the waveform does not return to baseline in the setting of an unstable spine, then continue with surgery, or if the spine is deemed stable, perform an awake clinical examination to determine whether to continue surgery. Ziewacz et al. ${ }^{22}$ recently proposed an evidence-based checklist (Fig. 2) for the response to significant IONM alerts in spine surgery. The effect of checklist usage during cervical spine surgery is currently being evaluated at our institution. First, however, the utility of IONM in cervical spine surgery must be established, ideally in the setting of a prospective randomized controlled clinical trial.

\section{Conclusions}

The use of IONM is increasing in cervical spine surgery. The findings from this study demonstrated a correlation between a decrease in intraoperative MEPs and new postoperative neurological deficits in cervical or cervicothoracic myelopathy cases. The performance of MEP monitoring as a diagnostic test varies based on risk factors such as patient comorbidities, age, and preoperative neurological function. We found that the sensitivity of MEP monitoring is higher in certain subgroups. Specifically, the sensitivity is increased in patients without vascular risk factors. Sensitivity is also increased in those aged 65 years and older as well as those with preoperative neurological deficits.

\section{Disclosure}

Dr. Chou reports being a consultant for Globus, DePuy, Medtronic, and Orthofix. Dr. Ames reports being a consultant for DePuy, Medtronic, and Stryker; holding a patent with Fish \& Richardson, P.C.; grants/pending grants from and stock/stock options in Trans1; receiving royalties from LANX and Aesculap; and having stock/stock options in Visualase and Doctors Research Group. Dr. Mummaneni reports honoraria and royalties from DePuy Spine, honoraria from Globus, and royalties from Thieme Medical Publishers and Quality Medical Publishing.

Author contributions to the study and manuscript preparation include the following. Conception and design: Mummaneni, Clark, Lyon. Acquisition of data: Mummaneni, Clark, Safaee, Lyon, Chou, Weinstein, Ames, Clark. Analysis and interpretation of data: Mummaneni, Clark, Ziewacz, Safaee, Lyon, Clark. Drafting the article: Mummaneni, Clark, Ziewacz, Clark. Critically revising the article: Mummaneni, Clark, Ziewacz, Safaee, Lau, Chou, Weinstein, Ames. Reviewed submitted version of manuscript: all authors. Statistical analysis: Mummaneni, Clark, Ziewacz. Administrative/technical/ material support: Mummaneni, Chou, Weinstein, Ames, Clark. Study supervision: Mummaneni.

\section{References}

1. Bose B, Sestokas AK, Schwartz DM: Neurophysiological monitoring of spinal cord function during instrumented anterior cervical fusion. Spine J 4:202-207, 2004

2. Costa P, Bruno A, Bonzanino M, Massaro F, Caruso L, Vincenzo I, et al: Somatosensory- and motor-evoked potential monitoring during spine and spinal cord surgery. Spinal Cord 45:86-91, 2007

3. Devlin VJ, Anderson PA, Schwartz DM, Vaughan R: Intraoperative neurophysiologic monitoring: focus on cervical myelopathy and related issues. Spine J 6 (6 Suppl):212S-224S, 2006

4. Eggspuehler A, Sutter MA, Grob D, Jeszenszky D, Porchet F, Dvorak J: Multimodal intraoperative monitoring (MIOM) during cervical spine surgical procedures in 246 patients. Eur Spine J 16 (Suppl 2):S209-S215, 2007

5. Epstein NE, Danto J, Nardi D: Evaluation of intraoperative somatosensory-evoked potential monitoring during 100 cervical operations. Spine (Phila Pa 1976) 18:737-747, 1993

6. Fan D, Schwartz DM, Vaccaro AR, Hilibrand AS, Albert TJ: Intraoperative neurophysiologic detection of iatrogenic C5 nerve root injury during laminectomy for cervical compression myelopathy. Spine (Phila Pa 1976) 27:2499-2502, 2002

7. Fehlings MG, Brodke DS, Norvell DC, Dettori JR: The evidence for intraoperative neurophysiological monitoring in spine surgery: does it make a difference? Spine (Phila Pa 1976) 35:S37-S46, 2010

8. Gandhoke G, Wu JC, Rowland NC, Meyer SA, Gupta C, Mummaneni PV: Anterior corpectomy versus posterior laminoplasty: is the risk of postoperative C-5 palsy different? Neurosurg Focus 31(4):E12, 2011

9. Gokaslan ZL, Samudrala S, Deletis V, Wildrick DM, Cooper PR: Intraoperative monitoring of spinal cord function using motor evoked potentials via transcutaneous epidural electrode during anterior cervical spinal surgery. J Spinal Disord 10:299-303, 1997

10. Hilibrand AS, Schwartz DM, Sethuraman V, Vaccaro AR, Albert TJ: Comparison of transcranial electric motor and somato- 


\section{A. J. Clark et al.}

sensory evoked potential monitoring during cervical spine surgery. J Bone Joint Surg Am 86-A:1248-1253, 2004

11. Kelleher MO, Tan G, Sarjeant R, Fehlings MG: Predictive value of intraoperative neurophysiological monitoring during cervical spine surgery: a prospective analysis of 1055 consecutive patients. J Neurosurg Spine 8:215-221, 2008

12. Kim DH, Zaremski J, Kwon B, Jenis L, Woodard E, Bode R, et al: Risk factors for false positive transcranial motor evoked potential monitoring alerts during surgical treatment of cervical myelopathy. Spine (Phila Pa 1976) 32:3041-3046, 2007

13. Lall RR, Lall RR, Hauptman JS, Munoz C, Cybulski GR, Koski T, et al: Intraoperative neurophysiological monitoring in spine surgery: indications, efficacy, and role of the preoperative checklist. Neurosurg Focus 33(5):E10, 2012

14. Lee JY, Hilibrand AS, Lim MR, Zavatsky J, Zeiller S, Schwartz DM, et al: Characterization of neurophysiologic alerts during anterior cervical spine surgery. Spine (Phila Pa 1976) 31:1916-1922, 2006

15. Meyer SA, Wu JC, Mummaneni PV: Laminoplasty outcomes: is there a difference between patients with degenerative stenosis and those with ossification of the posterior longitudinal ligament? Neurosurg Focus 30(3):E9, 2011

16. Ney JP, van der Goes DN, Watanabe JH: Cost-effectiveness of intraoperative neurophysiological monitoring for spinal surgeries: beginning steps. Clin Neurophysiol 123:1705-1707, 2012

17. Papastefanou SL, Henderson LM, Smith NJ, Hamilton A Webb JK: Surface electrode somatosensory-evoked potentials in spinal surgery: implications for indications and practice. Spine (Phila Pa 1976) 25:2467-2472, 2000

18. Schwartz DM, Sestokas AK, Turner LA, Morledge DE, DiNardo AA Jr, Beacham SG: Neurophysiological identification of iatrogenic neural injury during complex spine surgery. Semin Spine Surg 10:242-251, 1998

19. Smith PN, Balzer JR, Khan MH, Davis RA, Crammond D, Welch WC, et al: Intraoperative somatosensory evoked potential monitoring during anterior cervical discectomy and fusion in nonmyelopathic patients-a review of 1,039 cases. Spine J 7:83-87, 2007

20. Traynelis VC, Abode-Iyamah KO, Leick KM, Bender SM, Greenlee JD: Cervical decompression and reconstruction without intraoperative neurophysiological monitoring. Clinical article. J Neurosurg Spine 16:107-113, 2012

21. Xu R, Ritzl EK, Sait M, Sciubba DM, Wolinsky JP, Witham $\mathrm{TF}$, et al: A role for motor and somatosensory evoked potentials during anterior cervical discectomy and fusion for patients without myelopathy: analysis of 57 consecutive cases. Surg Neurol Int 2:133, 2011

22. Ziewacz JE, Berven SH, Mummaneni VP, Tu TH, Akinbo OC, Lyon R, et al: The design, development, and implementation of a checklist for intraoperative neuromonitoring changes. Neurosurg Focus 33(5):E11, 2012

Manuscript submitted March 15, 2013.

Accepted April 10, 2013.

Please include this information when citing this paper: DOI: 10.3171/2013.4.FOCUS13121.

Address correspondence to: Praveen V. Mummaneni, M.D., Department of Neurological Surgery, University of California, San Francisco, 505 Parnassus Ave., Rm. M779, San Francisco, CA 94143-0112. email: mummanenip@ neurosurg.ucsf.edu. 